\title{
The future of brown adipose tissues in the treatment of type 2 diabetes
}

\author{
Patrick Schrauwen $^{1}$ - Wouter D. van Marken Lichtenbelt ${ }^{1} \cdot$ Bruce M. Spiegelman $^{2}$
}

Received: 6 February 2015 / Accepted: 10 March 2015 / Published online: 9 May 2015

(C) Springer-Verlag Berlin Heidelberg 2015

\begin{abstract}
The recent recognition that humans possess active depots of brown adipose tissue has boosted the interest in this tissue as a potential target for the prevention and treatment of obesity and related metabolic disorders. Furthermore, it was also revealed that brown adipose tissue (BAT) in humans may consist of so-called beige or brite adipocytes. So far, cold exposure is recognised as the strongest activator of BAT in humans, but there is much ongoing research focused on finding alternative activators of BAT. The consequences of longterm BAT activation and/or cold exposure on metabolic health are still unknown, and this represents an area of intensive research. This is one of a series of commentaries under the banner ' 50 years forward', giving personal opinions on future perspectives in diabetes, to celebrate the 50th anniversary of Diabetologia (1965-2015).
\end{abstract}

Keywords Brown adipose tissue $\cdot$ Cold-induced thermogenesis $\cdot$ Diabetes $\cdot$ Energy turnover

\begin{abstract}
Abbreviations
BAT Brown adipose tissue

DNP Dinitrophenol
\end{abstract}

Patrick Schrauwen

p.schrauwen@maastrichtuniversity.nl

1 Department of Human Biology and Human Movement Sciences, NUTRIM School for Nutrition and Translational Research in Metabolism, Maastricht University Medical Center, PO Box 616, 6200MD Maastricht, the Netherlands

2 Dana-Farber Cancer Institute and Department of Cell Biology, Harvard Medical School, Boston, MA, USA
PET/CT Positron emission tomography-computed tomography

UCP1 Uncoupling protein-1

It was over 400 years ago that Conrad Gesner wrote about a fat depot in the neck of marmots that could be called neither fat nor flesh, but something in between [1]. More than 50 years ago, Smith [2] first recognised the thermogenic capacity of brown adipose tissue (BAT). This observation was followed in 1978 by the identification of uncoupling protein-1 (UCP1) as the principal component of brown adipose mitochondria responsible for regulating energy dissipation [3]. Subsequently, BAT was found to play an important role in the regulation of cold- and diet-induced thermogenesis, and revealed to be involved in body weight regulation in rodents [4]. In 2009, following retrospective patient studies, dedicated cold exposure studies using positron emission tomography-computed tomography (PET/CT) imaging revealed functional 'BAT' in humans, and confirmed the presence of increased levels of UCP1 in this tissue, resulting in the general acceptance that humans also possess active BAT stores [5-8]. In contrast to rodents, the 'BAT' depot in humans may consist of both classical brown adipocytes and brown-like adipocytes dispersed in white adipose tissue, but will be referred to as human BAT in the remainder of this commentary.

The last 50 years have been characterised by a dramatic rise in the prevalence of obesity and type 2 diabetes. Research initially focused on the regulation of energy expenditure as a crucial factor in the development of obesity, and it was indeed recognised that a relatively low resting or $24 \mathrm{~h}$ energy expenditure predisposes individuals to the development of obesity [9]. Mitochondrial energy dissipation via uncoupling was therefore considered a target for obesity treatment. However, the serious side effects of the uncontrolled use of the chemical 
uncoupler dinitrophenol (DNP), including hyperthermia and even death, in the $1930 \mathrm{~s}$ indicated that mitochondrial uncoupling should be induced in a very regulated and controlled manner, which diminished enthusiasm for mitochondrial uncoupling as a target for pharmacological intervention at that time. Although studies in the late 1990s revealed that UCP1 toxigene-mediated ablations of BAT resulted in the development of obesity and diabetes at ambient temperatures [4], the definitive identification of BAT in adult humans in 2009 really boosted hopes that enhancing energy expenditure may be a realistic option for the prevention and treatment of obesity-associated metabolic disorders. Indeed, studies conducted in the last 5 years have hinted towards novel ways of activating human BAT and have revealed that BAT activity may indeed be related to energy expenditure (for review, see [10]).

\section{Energy turnover as a tool to combat obesity-related metabolic disorders?}

So can human BAT contribute to a solution to the metabolic health problems that we are currently facing? Although the idea that activating BAT will be the complete answer to a large societal problem of habitual overeating and physical inactivity is certainly oversimplifying things, there are positive reasons to hope that the next 50 years will reveal major breakthroughs in our understanding of how stimulating (brown) adipose tissue and energy turnover may promote metabolic health.

First of all, the concept of energy balance as the most important factor in determining metabolic health will have to be revisited. Instead, a high rate of energy and substrate turnover may be crucial. Indeed, exercise and physical activity do not usually result in negative energy balance or weight loss but are important for maintaining metabolic health. Also, at the cellular level, the turnover of chemical energy and substrate seems to determine cellular function. For example, the development of insulin resistance and type 2 diabetes has been blamed, at least in part, on ectopic fat accumulation in muscle, liver and heart: consistent with the 'energy balance' concept, a low mitochondrial oxidative capacity has subsequently been proposed to underlie the fat accumulation in these tissues [11]. However, more recent findings suggest that a high — or stimulated-mitochondrial function does prevent cellular lipotoxicity, but not by lowering ectopic fat accumulation [12]. To date, the exact mechanisms underlying these effects are not understood, but the data suggest that the continuous storage and breakdown of energy stores can prevent the negative consequences of excess energy storage. Over the next 50 years, we will learn if and how the dissipation of energy in any given tissue can increase whole body energy fluxes and may thereby promote cellular function and metabolic health. Among the things we need to determine is how increasing energy flux in one tissue can lead to effects in other tissues - a field that is still in its infancy and was only initiated some 20 years ago with the discovery that adipose tissue also has an endocrine function and a role in inter-organ communication. The list of inter-organ signals secreted by white, brown and beige adipocytes, muscle, liver and other tissues will continue to increase in the coming years, and important knowledge on the complex system of inter-organ communication will aid the development of targets for tissue-specific manipulation of energy turnover.

\section{The novel functions of fat, fat and fat}

The discovery of human BAT will fuel a new line of research dedicated to understanding the precise physiological function of this tissue. For this, we first need a better understanding of the anatomical locations of BAT in humans. In rodents, beige fat is dispersed, and with the current techniques used to detect BAT in humans we may only see tight depots and underestimate its full potential. We also need to reveal whether BAT 'only' plays a role in thermoregulation or if this tissue has other, yet unrevealed, functions. BAT activation requires fatty acids and glucose as a fuel, and experimental evidence in rodents suggests that BAT may be involved in the clearance of glucose and triacylglycerols from the circulation. It is unknown whether this is just a side effect of the (thermogenic) activity of BAT or a dedicated and controlled function of this tissue, nor is it known whether activation of BAT in humans indeed can contribute to the regulation of circulating glucose and triacylglycerol levels and thereby exert cardioprotective and anti-diabetic effects, but data from animal studies are promising $[13,14]$. In fact, we are currently still facing experimental limitations in detecting active BAT in humans, and the gold standard so far-cold-induced glucose uptake using 2-deoxy-2- $\left[{ }^{18} \mathrm{~F}\right]$ fluoro-D-glucose $\left(\left[{ }^{18} \mathrm{~F}\right] \mathrm{FDG}-\mathrm{PET} / \mathrm{CT}\right)$ - may only reveal part of the activity of human BAT, and novel, non-invasive techniques are warranted to study the metabolism of BAT in humans in vivo in more detail.

Perhaps an even more promising 'side effect' of the current renewed interest in the study of BAT is the recognition that not all fat is the same. Whereas approximately 20 years ago, white adipose tissue was considered a boring tissue serving mainly as a large energy reservoir, the discovery of several adipokines in the 1990s added a new dimension to the tissue as one of endocrine origin. However, even nowadays, most people, including health scientists and medical doctors, consider white adipose tissue to be one single, albeit very large, organ, perhaps with visceral adipose tissue considered as a special kind of metabolically active fat. It is realistic to expect that the next 50 years will reveal that this is far from the truth. The study of BAT has already revealed a new distinct type of thermogenic fat cells located in white adipose tissue depots, termed beige or 
brite adipocytes, and their function has only just started to be unravelled (Fig. 1). In fact, data now suggest that a major portion of BAT in humans is not composed of classical brown fat cells, as in the interscapular depots of rodents, but are composed of beige cells [15]. It is clear that beige fat contributes to the metabolic health of genetically defined mouse models [16], but how human beige fat cells contribute to energy metabolism needs further study. Also, we need to establish the specific and distinct physiological functions of brown vs beige adipocytes. With the rapidly increasing developments in molecular biology, new tools will become available that are likely to result in the discovery of even more adipose tissue cell types or subtypes and their associated functions. These all providing new challenges to understanding the complex system of metabolism, but also presenting new opportunities for the development of finely tuned therapies for the treatment of metabolic diseases.

\section{Towards new therapies for diabetes and metabolic complications of obesity}

The discovery of new types of adipose cells, new adipokines and new biological functions of tissues containing these cells could provide many new potential therapies for the treatment of obesity, diabetes and related disorders. The list of targets that may induce browning or activate beige/brown adipose tissue is expanding daily, and research in the coming years will reveal which of these are robust enough to represent new opportunities for human therapy. Several key questions arise: first, is it more important to create new brown or beige fat cells or to activate existing one in an individual, or do we need to do both? Second, many studies in rodents have revealed beneficial effects on glucose homeostasis and diabetes, which may not be in proportions with effects on body weight per se. Are there important effects of brown/beige fat that are not strictly tied to effects on overall adiposity? It might be foolish to place all our hope on novel pharmacological treatment. It will remain very important that public health initiatives stress the health dangers of physical inactivity and overeating; the coming years will also reveal whether a lack of time outside the thermoneutral zone should be considered an additional danger to health. Based on the results to date, it can be hypothesised that regular exposure to mild cold may become - together with exercise and weight loss - a third lifestyle factor that can be used to stimulate health [17]. As with lifestyle and exercise, we need to understand how much cold is enough; are we facing a ' 30 min of cold per day keeps the doctor away' advice, or will $30 \mathrm{~min}$ of cold per day not be sufficient to counterbalance being in the thermoneutral zone

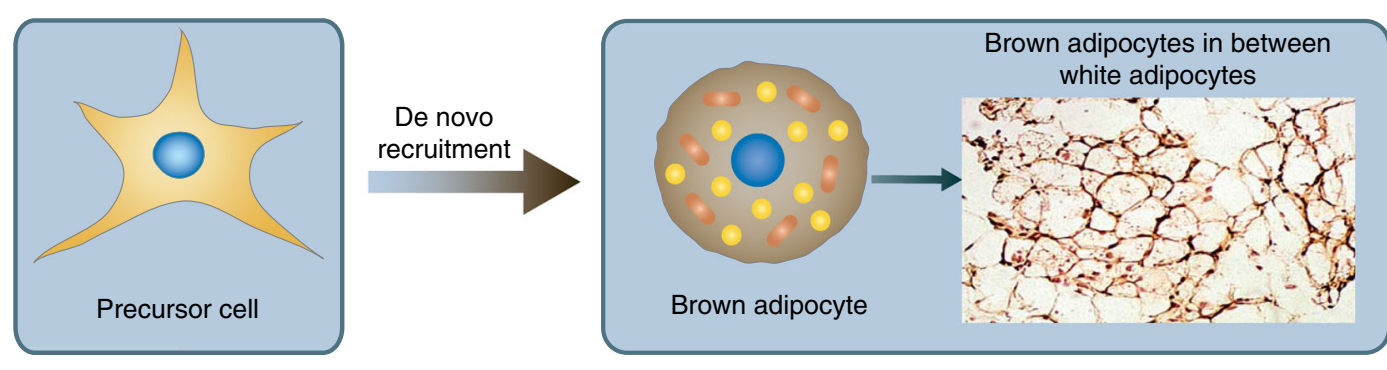

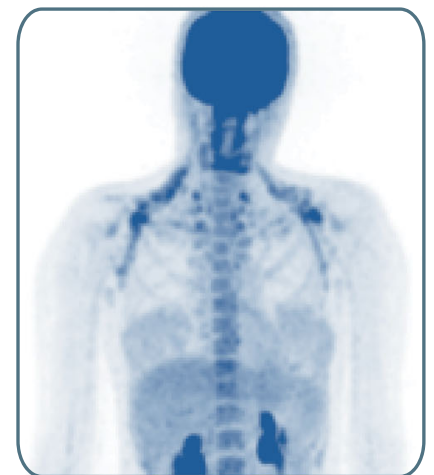

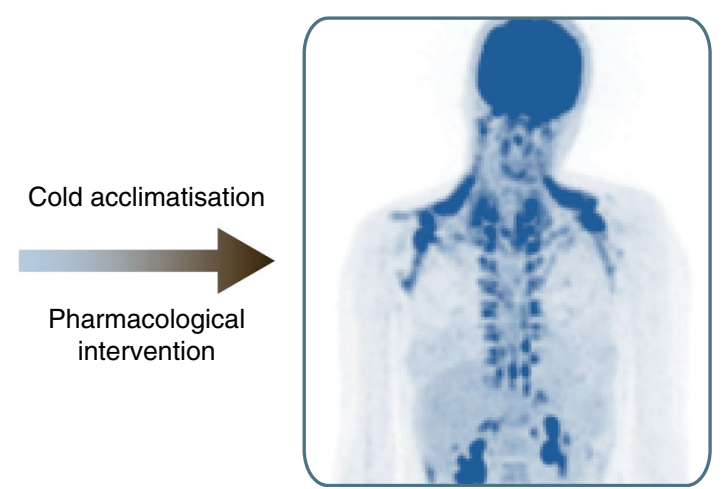

Fig. 1 BAT contains high levels of UCP1, which allows it to perform its major role of dissipating energy as heat. Cold acclimatisation and some pharmacological agents have been shown to cause activation of BAT or browning of white adipose tissue in adult humans, as indicated by the darker areas on the $\left[{ }^{18} \mathrm{~F}\right]$ FDG-PET/CT scans. As depicted in the illustration, the brown cells may originate from de novo recruitment of precursor cells. The extent to which browning is accomplished by classical brown adipocytes or the recruitment of beige cells in humans is still not completely understood. Studies to date indicate that in humans the tissue is heterogeneous with a mix of brown (beige) and white adipocytes, as shown in the micrograph. As currently available techniques are not sensitive enough to detect diffuse BAT, the precise anatomical location of browning is worthy of more in-depth study. It is hoped that further developments in this field will reveal novel methods for the prevention and treatment of obesity-associated metabolic disorders 
for $23.5 \mathrm{~h}$ per day, analogous to $30 \mathrm{~min}$ of exercise per day perhaps not being sufficient to counterbalance $23.5 \mathrm{~h}$ of sedentariness? The cold acclimatisation tests carried out to date have lasted 2-6 h/day for 10 days to 6 weeks, with temperatures varying from very cold $\left(10^{\circ} \mathrm{C}\right.$ with shivering) to mild cold $\left(19^{\circ} \mathrm{C}\right)$. Even mild cold (without shivering) increases BAT activity, whole body energy metabolism and decreases body fat, but long-term effects on measures of health still need to be established, and optimal exposure times, i.e. a compromise between realistic and effective, will need to be figured out. Furthermore, it needs to be established whether the potential beneficial effects of cold are only due to the activation of BAT, or also involve other tissues with a thermogenic potential, such as skeletal muscle. Cooling protocols would need to be developed taking into account the large variability in individual and group-specific responses with respect to effects on BAT and energy metabolism and in terms of acceptability, i. e. thermal comfort. These group-specific responses also apply to potential side effects, as, for example, the elderly may not respond adequately to prolonged mild cold and their blood pressure may rise to unhealthy levels.

An interesting aspect of cold 'therapy' is that it can be incorporated into our daily environment, i.e. the indoor environment. Because most people are exposed to indoor conditions for more than $90 \%$ of their time, health aspects of ambient temperatures warrant exploration. What would it mean if we were to let our bodies, rather than the central heating, control body temperature? Even if the effect at an individual level is small, with widespread application in dwellings and offices, the effect on the population could be significant. In the built environment the temperature is controlled to satisfy thermal comfort for the average (male) person. This is according to the predicted mean vote model that is generally applied worldwide. This results in relatively high temperatures in wintertime. By lack of exposure to a varied ambient temperature, entire populations may be prone to developing diseases such as obesity and in the same time may become more vulnerable to sudden temperature changes during cold waves. Nowadays the so-called adaptive comfort model is more widely applied. This model recognises that humans tolerate and adapt to different thermal environmental conditions depending on outdoor environmental conditions. For the next 50 years, we envision that indoor temperature will be used to enhance metabolism and resilience, and thereby health in general. Although scientific attention is now focused on cold, the health aspects of mild heat are still an enigma and need to be explored. Therefore the next 50 years will unravel whether, from a health perspective, indoor temperatures should follow outdoor temperature variation at acceptable but healthy levels, both daily and seasonally.
Duality of interest The authors declare that there is no duality of interest associated with this manuscript.

Contribution statement All authors were responsible for drafting the article and revising it critically for important intellectual content. All authors approved the version to be published.

\section{References}

1. Gesner C (1551) Historiæ animalium lib. I. Tigvri, Apvd Christ Froschovervm (in Latin)

2. Smith RE (1961) Thermogenic activity of the hibernating gland in the cold-acclimatized rat. Physiologist 4:113

3. Nicholls DG, Bernson VS, Heaton GM (1978) The identification of the component in the inner membrane of brown adipose tissue mitochondria responsible for regulating energy dissipation. Experientia Suppl 32:89-93

4. Lowell BB, S-Susulic V, Hamann A et al (1993) Development of obesity in transgenic mice after genetic ablation of brown adipose tissue. Nature 366:740-742

5. Cypess AM, Lehman S, Williams G et al (2009) Identification and importance of brown adipose tissue in adult humans. N Engl J Med 360:1509-1517

6. van Marken Lichtenbelt WD, Vanhommerig JW, Smulders NM et al (2009) Cold-activated brown adipose tissue in healthy men. N Engl J Med 360:1500-1508

7. Virtanen KA, Lidell ME, Orava J et al (2009) Functional brown adipose tissue in healthy adults. N Engl J Med 360:1518-1525

8. Saito M, Okamatsu-Ogura Y, Matsushita M et al (2009) High incidence of metabolically active brown adipose tissue in healthy adult humans: effects of cold exposure and adiposity. Diabetes 58:15261531

9. Ravussin E, Lillioja S, Knowler WC et al (1988) Reduced rate of energy expenditure as a risk factor for body-weight gain. N Engl J Med 318:467-472

10. Vosselman MJ, van Marken Lichtenbelt WD, Schrauwen P (2013) Energy dissipation in brown adipose tissue: from mice to men. $\mathrm{Mol}$ Cell Endocrinol 379:43-50

11. Petersen KF, Dufour S, Befroy D, Garcia R, Shulman GI (2004) Impaired mitochondrial activity in the insulin-resistant offspring of patients with type 2 diabetes. N Engl J Med 350:664-671

12. Phielix E, Meex R, Ouwens DM et al (2012) High oxidative capacity due to chronic exercise training attenuates lipid-induced insulin resistance. Diabetes 61:2472-2478

13. Bartelt A, Bruns OT, Reimer R et al (2011) Brown adipose tissue activity controls triglyceride clearance. Nat Med 17:200-205

14. Labbè SM, Caron A, Bakan I et al (2015) In vivo measurement of energy substrate contribution to cold-induced brown adipose tissue thermogenesis. FASEB J. doi:10.1096/fj.14-266247

15. Wu J, Bostrom P, Sparks LM et al (2012) Beige adipocytes are a distinct type of thermogenic fat cell in mouse and human. Cell 150: 366-376

16. Cohen P, Levy JD, Zhang Y et al (2014) Ablation of PRDM16 and beige adipose causes metabolic dysfunction and a subcutaneous to visceral fat switch. Cell 156:304-316

17. Lichtenbelt W, Kingma B, van der Lans A, Schellen L (2014) Cold exposure-an approach to increasing energy expenditure in humans. Trends Endocrinol Metab 25:165-167 